\title{
Pre-anesthesia clinic: skip it or not? A case report
}

\author{
Abdullah King Hamad \\ University Hospital, \\ Shaikh Isa Bin Salman \\ Causeway, Al Sayh, Bahrain \\ Correspondence: Dr. Amr \\ Abdullah King Hamad \\ University Hospital, Shaikh \\ Isa Bin Salman Causeway, Al \\ Sayh, Bahrain; \\ Phone: +973 36553550; \\ E-mail: amr.abdullah@khuh. \\ org.bh \\ Received: 3 April 2019, \\ Reviewed: 7 May 2019, \\ Accepted: 27 May 2019
}

\author{
Amr Abdullah, MD, Amir Fouad, MSc, FEBA, CABA-IC, DESA, \\ Ahmed Mamdouh Esmat, MSc, FEBA, CABA-IC, \\ Ali Elhefnawy, MSc, FEBA, CABA-IC, DESA, FCAI, \\ Shahid Adeel, FCAI
}

\section{INTRODUCTION}

The preoperative assessment clinic should be used for preoperative evaluation of patients, allowing the anesthetist to decide the most suitable modality of anesthesia to be administered intraoperatively, increasing the safety of anesthesia, and explaining to patients the expected outcomes from anesthesia and addressing any fears they may have. This reduces the total number of surgeries cancelled and increases patient satisfaction ${ }^{(1)}$. The ultimate goals of preoperative assessment are to assess perioperative risk, improve patient status and to decrease the patient's surgical and anesthetic morbidity or mortality (2).

The aim of this case presentation is to highlight the significance of preoperative anesthesia assessment before surgery, as proper and thorough assessment may be helpful in identifying conditions, which previously may have remained undiagnosed and untreated.

\section{CASE REPORT}

We present a case of a 64-year-old male who presented to our pre-anesthesia clinic for assessment prior to an elective right total knee replacement procedure. He had a past medical history of hypertension, type II diabetes mellitus, dyslipidemia, ischemic heart disease, and a history of coronary angioplasty with stenting for an inferior wall myocardial infarction that was performed in 2007.

The patient was taking a number of prescription medication on a regular basis, such as: diamicron (gliclazide), glucophage (metformin), norvasc (amlodipine), concor (bisoprolol), aspirin, preterax (perindopril arginine/indapamide), and zocor (simvastatin).

On examination, the patient was found to be vitally stable, with a heart rate of $84 \mathrm{bpm}$, a blood pressure of $114 / 67 \mathrm{mmHg}$, and an oxygen saturation of $100 \%$ on room air. He was conscious, oriented, no pallor or pedal edema. He was previously physically active and 
Figure 1: Image of ECG done at pre-anesthesia clinic visit

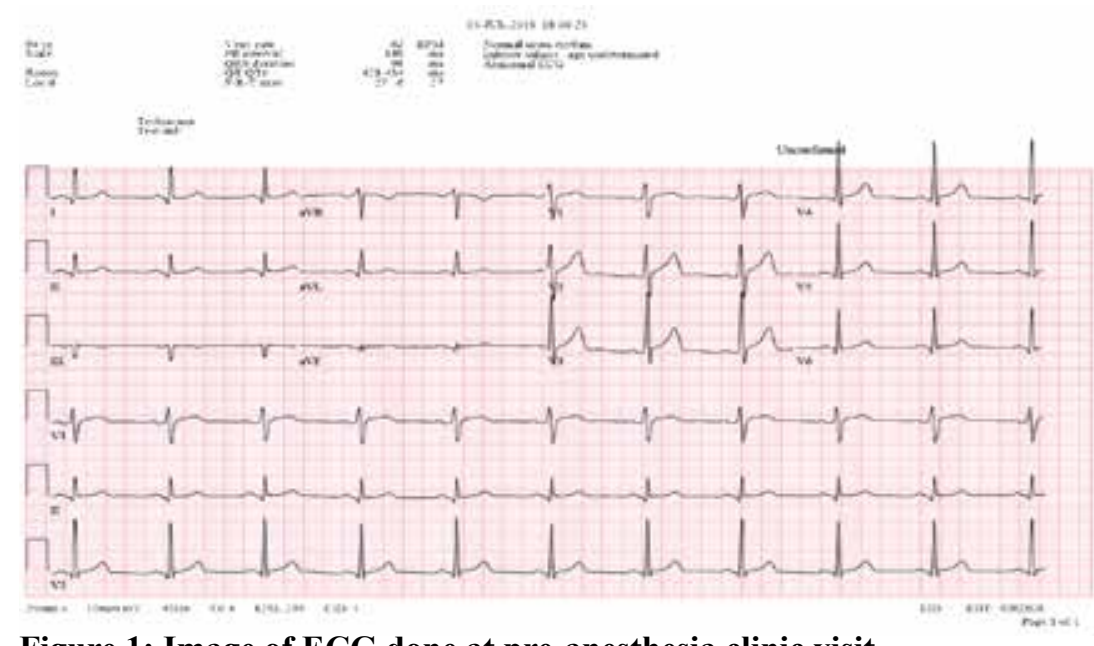

had a previous history of ischemic heart disease with angioplasty and there was no ECHO in the patient records. Findings showed a normal left ventricular cavity size with a normal global systolic function ejection fraction of $60 \%$. There was normal resting wall motion. A grade 1 left ventricular diastolic dysfunction was apparent, along with sclerotic mitral leaflets, trivial mitral regurgitation and an intact pericardium, which were all typical for the patient's age and history. An incidental finding of a sclerotic aortic valve with highly mobile mass was seen attached to the right coronary cusp on the aortic side with same echogenicity

with no cardiac symptoms. The patient had no chest pain, shortness of breathing, or palpitations.

S1 and S2 heart sounds were regular on auscultation, there were no audible murmurs and his chest was clear with bilaterally equal air entry. Lower limb examination was negative, there was no lower limb edema and distal pulses were felt with a regular rhythm.

The only complaint the patient had was chronic bilateral knee pain which was worse on the right when compared to the left. The pain was aggravated on walking or climbing up stairs. Prior to the onset of said pain, the patient was able to walk for 30 to 60 mins without any issues as well as climb at least 2 flights of stairs without feeling out of breath. An ECG was done which showed a regular sinus rhythm with an inferior infarct of undetermined age.

An ECHO was ordered on the basis that the patient

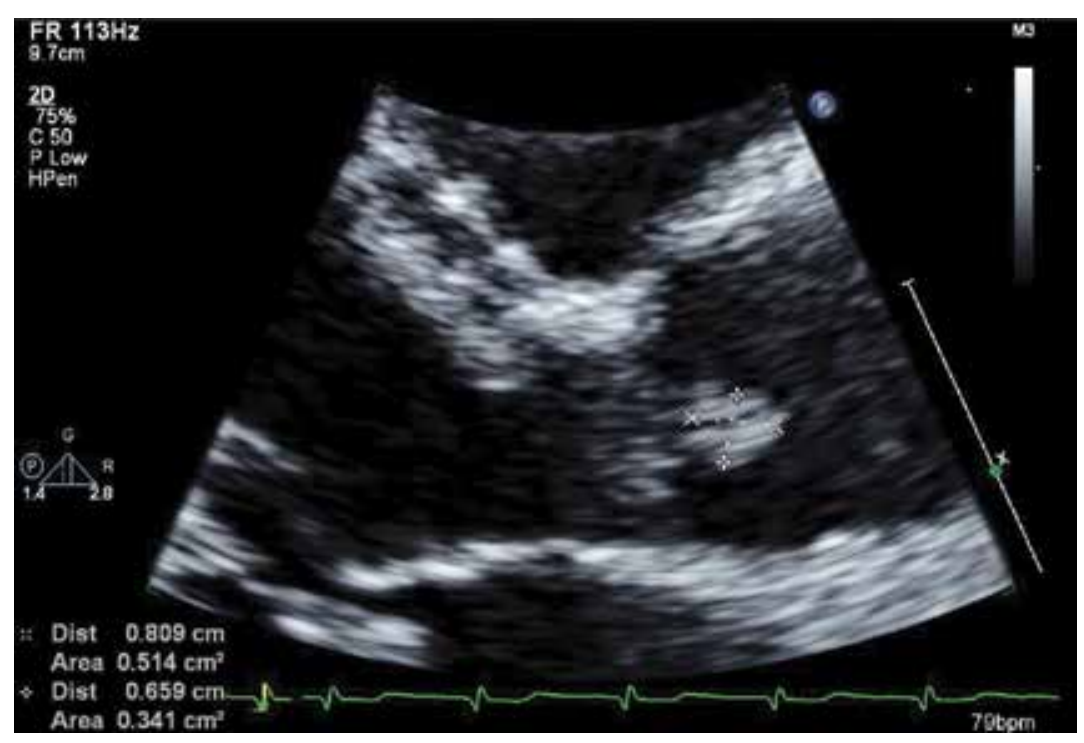

Figure 2: Image of ECHO with highly mobile mass as the valve, no aortic regurgitation, no significant systolic gradient. The report advised for a differential diagnosis and further evaluation.

The patient was referred for a cardiology consultation and was then referred to the cardiac center for further evaluation. Later on, he was transported to the cardiac center along with copies of all documents and investigations.

After a period of four months the patient returned to the pre-anesthesia clinic for assessment for the same procedure. He had undergone a resection of the papillary fibroelastoma of the aortic valve 3 months ago, which included coronary artery bypass grafting (CABG) of 3 arteries: the left anterior descending (LAD), the obtuse marginal artery 1 (OMA 1), and the obtuse marginal artery 3 (OMA 3).

During his visit to the clinic he was doing well with no cardiac symptoms. He was conscious, oriented, no pallor or pedal edema. Vitals were within normal range; a pulse of $90 / \mathrm{min}$, a blood pressure of $110 / 70$ $\mathrm{mm} \mathrm{Hg}$ with normal heart sounds. $\mathrm{He}$ was maintaining an oxygen saturation of $96 \%$ on room air.

Post-operative ECHO showed to have normal left ventricular dimensions with post-op septal motion pattern and sluggish basal inferior segment, overall systolic function of $50 \%-55 \%$ and grade 1 diastolic dysfunction. Sclerotic mitral valve leaflets with mild regurgitation. Sclerotic aortic valve with no significant systolic gradient nor regurgitation and an estimated systolic pulmonary artery pressure of $26 \mathrm{mmHg}$. Patient was advised to continue all 
his anti-hypertensive medications and discontinue the morning dose of the oral hypoglycemic.

The available anesthetic techniques for the procedure were fully explained to him, which included general and spinal anesthesia. He opted for spinal anesthesia.

Spinal anesthesia was performed using $2.8 \mathrm{~mL}$ of heavy bupivacaine $0.5 \%$ along with $25 \mathrm{mcg}$ of fentanyl using a midline approach in the sitting position at the level of L3-L4 with a sensory block level of T10. Prior to that he received $500 \mathrm{~mL}$ of ringer lactate $(\mathrm{RL})$ solution as a bolus infusion. $\mathrm{He}$ was stable throughout the procedure without any significant decline in blood pressure or heart rate. A total of 2 liters of RL was insfused along with 1 gram of paracetamol. $3 \mathrm{mg}$ of midazolam was given in total, in increments of $1 \mathrm{mg}$. He was also given 4 $\mathrm{mg}$ of ondansetron as prophylaxis against any postoperative nausea and vomiting. During the procedure the patient was also supplemented with 5 litres $/ \mathrm{min}$ of oxygen using a facemask. After the procedure the patient was shifted to the recovery station, where he was kept for 45 minutes under close observation and then finally shifted safely to the ward. His overall stay in the hospital remained uneventful.

\section{DISCUSSION}

The preoperative anesthetic assessment is a key component of the anesthetic management. The American Society of Anesthesiologists established guidelines describing that each patient requiring care by an anesthetist must be provided a comprehensive medical and anesthetic evaluation, which includes a full drug history, physical examination, proper diagnostic testing, assignment of an ASA physical status score, and formulation and discussion of an anesthetic plan. ${ }^{3}$ This practice allows for recognition of any disease processes that may alter perioperative anesthetic care and provide a preoperative risk stratification and sufficient preparation to provide safe and adequate anesthesia.

It is important to understand that "perioperative" risk is multifactorial which includes the preoperative medical condition of the patient, the invasiveness of the surgical procedure and the type of anesthetic administered. It is crucial to any preoperative evaluation that patient history be taken and a physical examination performed, with attention to risk factors for cardiac and pulmonary complications to determine a patient's functional capacity. Laboratory investigations should only be ordered when indicated by the patient's medical status, drug therapy, or the nature of the proposed procedure and not on a routine basis. ${ }^{2,4}$ These investigations are performed with the rational expectation that they will be beneficial to patient safety and improve anesthetic management, which would outweigh any potential inconvenience or delay caused.

There are many case reports of incidental findings that altered the patient's management, leading to favorable outcomes. In one of the published case reports, a 74-year-old female scheduled for an orthopedic procedure was found to have an atrial thrombus diagnosed by a preoperative transthoracic ECHO. She was promptly started on an anticoagulant regime for 5 months until the mass was gone. 5 In another case report an aortic valve Papillary Fibroelastoma (PFE) was coincidentally found on routine transesophageal ECHO in a patient booked for a myocardial revascularization procedure. This led to a modified approach to resect the tumor during the revascularization. ${ }^{6}$ Similarly, a case report has been published by Prifti E et al regarding incidental finding of Mitral valve PFE in a patient who presented with wound infection after Mastectomy. The tumor was subsequently excised, thus reducing the risk of embolization. ${ }^{7}$

PFEs are benign neoplasms of the valves of the heart, they represent roughly $10 \%$ of primary cardiac tumors and are the second most common benign neoplasms of the cardiac valves after myxomas. ${ }^{8-10}$ They are usually found in the elderly, with a mean age of 6070 years, but it can occur in any age group..$^{10}$ PFEs are commonly found incidentally on autopsy or as an intraoperative surgical finding.

They are also sometimes discovered during echocardiography or catheterization, ${ }^{8}$ with transoesophageal ECHO having a tendency to provide a clearer diagnosis. ${ }^{11}$

$85 \%$ of PFEs originate from the valvular endocardium, but can also be found on the papillary muscles, the endocardial surface, the ventricular septum, or the chordae tendenae. ${ }^{9} 29 \%$ of PFE's originating from the valvular endocardium are most commonly from the aortic valve, $25 \%$ from the mitral valve, $17 \%$ from the tricuspid valve, and $13 \%$ from pulmonary valves. ${ }^{12}$

Despite these tumors being benign in nature, if they are left unidentified, they can carry a very high risk of embolic complications and neurological deficits. ${ }^{13}$ Direct and indirect symptoms of PFEs can include syncope, angina pectoris, transient ischemic attack, stroke, pulmonary embolism, myocardial infarction, congestive heart failure, and sudden death. ${ }^{8}$ This is due to the nature of the papillary tissue of the tumor being fragile and pedunculated leading to them being susceptible to thromboembolism. Therefore, once a diagnosis has been made, urgent surgical management is indicated even in an asymptomatic patient. ${ }^{6,14}$

\section{CONCLUSION}

Comprehensive preoperative anesthetic assessment is essential to any patient scheduled for an elective 
procedure, as it may identify undiagnosed conditions that may have proven to be detrimental to the patient's perioperative care.

Conflict of Interest: None.

Authors contributions:

\section{REFERENCES}

1. Soares RR, Valadares FW, Araujo $M B$, Lorentz MN. Importance of preanesthetic evaluation: case report of a patient with obstructive sleep apnea. Rev Bras Anestesiol. 2011 NovDec;61(6):786-92. [PubMed] DOI: 10.1016/S0034-7094(11)70088-5

2. Zambouri A. Preoperative evaluation and preparation for anesthesia and surgery. Hippokratia. 2007 Jan;11(1):13-21. [PubMed]

3. Blitz JD, Kendale SM, Jain SK, Cuff GE, Kim JT, Rosenberg AD. Preoperative evaluation clinic visit is associated with decreased risk of in-hospital postoperative mortality. Anesthesiology. 2016 Aug;125(2):280-94. [PubMed] Dol: 10.1097/ALN.0000000000001193

4. Committee on Standards and Practice Parameters, Apfelbaum JL, Connis RT, Nickinovich DG, American Society of Anesthesiologists, Task Force on Preanesthesia Evaluation, Pasternak LR, et al. Practice advisory for preanesthesia evaluation: an updated report by the American Society of Anesthesiologists task force on preanesthesia evaluation. Anesthesiology. 2012 Mar;116(3):522-38. [PubMed] DOI: 10.1097/ALN.0b013e31823c1067

5. Harada M, Fujii Y, Tabako S, Takarada
$A A$ : manuscript writing, conduction of the case

AF: manuscript writing, literature review

AME: concept, literature review

AE: manuscript writing

SA: supervisor, manuscript editing
Y, Hayashi K, Hara F. A case of left atrial thrombus accidentally found by preoperative evaluation for orthopedic surgery using echocardiography. Choonpa igaku. 2015 Mar;42(2):165-70. DOl 10.3179/jjmu.JJMU.A.31

6. Gopaldas RR, Atluri PV, Blaustein AS, Bakaeen FG, Huh J, Chu D. Papillary fibroelastoma of the aortic valve-operative approaches upon incidental discovery. Tex Heart Inst J. 2009;36(2):160-3. [PubMed]

7. Prifti E, Ademaj F, Ikonomi M, Demiraj A. Papillary fibroelastoma of the anterior leaflet of the mitral valve mimicking vegetation. J Surg Case Rep. 2015 Jul 17;2015(7). [PubMed] DOI: 10.1093/jscr/rjv091

8. Raju V, Srinivasan M, Padmanaban C, Soundararajan S, Kuppanna PJ. Left main coronary artery embolus: unusual presentation of papillary fibroelastoma of the aortic valve. Tex Heart Inst J. 2010;37(3):365-7. [PubMed]

9. Czekajska-Chehab E, Tomaszewski A, Wójcik M, Drop A. Papillary fibroelastoma as an accidental finding in a multislice computed tomography scan of coronary arteries. Can $\mathrm{J}$ Cardiol. 2006 Nov;22(13): 1155-7. [PubMed]

10. Amano J, Nakayama J, Yoshimura
Y, Ikeda U. Clinical classification of cardiovascular tumors and tumorlike lesions, and its incidences. Gen Thorac Cardiovasc Surg. 2013 Mar;61(8):435-47. [PubMed]

11. Sun JP, Asher CR, Yang XS, Cheng GG, Scalia GM, Massed AG, et al. Clinical and echocardiographic characteristics of papillary fibroelastomas: a retrospective and prospective study in 162 patients. Circulation. 2001 Jun 5;103(22):2687-93. [PubMed]

12. Edwards $F H$, Hale $D$, Cohen $A$, Thompson L, Pezzella AT, Virmani R. Primary cardiac valve tumors. Ann Thorac Surg. 1991 Nov;52(5):112731. [PubMed]

13. Grinda JM, Couetil JP, Chauvaud $\mathrm{S}$, D'Attellis N, Berrebi A, Fabiani $\mathrm{JN}$, et al. Cardiac valve papillary fibroelastoma: surgical excision for revealed or potential embolization. J Thorac Cardiovasc Surg. 1999 Jan;117(1):106-10. [PubMed]

14. Domenech A, de Arenaza DP, Rivello HG, Patrucco L, Bracco D. Surgery for papillary fibroelastoma with uncommon location in left ventricle. Asian Cardiovasc Thorac Ann. 2010 Feb;18(2):174-6. [PubMed] DOl: 10.1177/0218492310361391 